\title{
Micro and nanotechnology for biological and biomedical applications
}

\author{
Chwee Teck Lim · Jongyoon Han · Jochen Guck • \\ Horacio Espinosa
}

Received: 28 August 2010/Accepted: 2 September 2010/Published online: 16 September 2010

(C) International Federation for Medical and Biological Engineering 2010

\begin{abstract}
This special issue contains some of the current state-of-the-art development and use of micro and nanotechnological tools, devices and techniques for both biological and biomedical research and applications. These include nanoparticles for bioimaging and biosensing, optical and biophotonic techniques for probing diseases at the nanoscale, micro and nano-fabricated tools for elucidating molecular mechanisms of mechanotransduction in cell and molecular biology and cell separation microdevices and techniques for isolating and enriching targeted cells for disease detection and diagnosis. Although some of these works are still at the research stage, there is no doubt that some of the important outcomes will eventually see actual biomedical applications in the not too distant future.
\end{abstract}

\section{T. $\operatorname{Lim}(\bowtie)$}

Mechanobiology Institute, Division of Bioengineering

and Department of Mechanical Engineering, National University

of Singapore, Singapore 117576, Singapore

e-mail: ctlim@nus.edu.sg

J. Han

BioSystems and Micromechanics (BioSyM) IRG, SingaporeMIT Alliance for Research and Technology (SMART) Centre, Singapore 117543, Singapore

J. Han

Department of Electrical Engineering, Computer Science and Biological Engineering, Massachusetts Institute of Technology, Cambridge, MA 02139, USA

J. Guck

Cavendish Lab, Department of Physics, University of Cambridge, Cambridge CB3 OHE, UK

H. Espinosa

Department of Mechanical Engineering, Northwestern University, Evanston, IL 60208-3111, USA
The last decade has seen increasing use of micro and nanotechnology for biological and biomedical applications. This can be attributed to several reasons. Firstly, there is tremendous advancement in the research and development of micro and nanotechnological tools and devices. Secondly, much progress in cell and molecular biology and biomedical sciences has also been made. Thirdly, there is a huge push towards multidisciplinary research that involves close collaboration among researchers from several disciplines including physics, chemistry, engineering, biology, and medicine. In fact, it is through this collaboration that we are starting to see how micro and nanotechnology are catalyzing and even accelerating research in the biological and biomedical sciences. Over the last 100 years, technology has always been seen to play an important contributing role in advancing biological and biomedical research and to its eventual applications in the clinics and hospitals. Thus, it is not surprising for micro and nanotechnology to play the same role and make similar contributions as well.

The development of micro and nanotechnological tools and devices can now enable us to image and probe cells and biomolecules that were previously impossible. We can now easily characterize the minute responses of individual cells and biomolecules in the micro, nano, and even picoscale range when subjected to mechanical, electrical, magnetic, and/or biochemical stimuli. In fact, micro and nanotechnology do offer several advantages over current conventional macroscale technology for any such test, experiment or application. These advantages include: (i) only a minute amount of sample and testing agents are needed; (ii) fast processing time as analysis time is reduced due to minute amount of sample being tested; (iii) highly sensitive and increased accuracy; (iv) increased portability, especially for biomedical application where there is 
potential for point-of-care diagnostic in the form of a microchip in resource poor settings that may lack clinical labs and skilled personnel; and (vi) low cost [6, 9, 20, 21]. Arising from these advantages, there is a unique opportunity for such micro and nanotechnological tools and devices to be further developed from bench to eventual biomedical applications at the bedside.

This special issue highlights some of the current stateof-the-art research being done around the world in the development or use of micro and nanotools, devices and techniques for biological and biomedical research and applications.

One of the hotly pursued nanotechnology research is in the fabrication and use of nanoparticles. Nanoparticles, having sizes less than $100 \mathrm{~nm}$, are at the same scale as proteins and biomolecules. In this issue, Nagarajan et al. [16] demonstrated the application of upconversion nanoparticles to target the gap junction formed between cardiac cells. As the particles do not enter the cells but target the proteins expressed at the surface of the gap junctions or on the cell membrane, it has great potential in bioimaging living cells without subjecting the cells to any adverse effects.

The last decade has seen an enormous surge made in research on biomagnetism and magnetic biosensors based on molecular recognition processes. In fact, there has been increasing use of magnetic nanoparticles for applications in biomedicine [17]. Here, Llandro et al. [13] first presented conventional methods of performing biological assays and molecular identification, highlighted their advantages and limitations, and then presented an alternative approach based on magnetic nanotechnology.

Optical and biophotonic techniques are some of the important methods that one can use to image and reveal biological structures at the micro and nanoscale. Mauritz et al. [14] reviewed a range of optical and biophotonic techniques developed to make quantitative measurements of malaria-infected blood cells. These included measuring haemoglobin concentration by fluorescence lifetime imaging and diffractive phase microscopy, cell volumes by quantitative deconvolution microscopy and ion content by $\mathrm{X}$-ray analysis methods. They also described how nanotools such as optical tweezers and optical stretchers can be used to shed light on the detailed molecular mechanisms of malaria infection that lead to the pathophysiology of the disease with the hope of finding new diagnostic or therapeutic approaches.

Several articles focused on the use of micro and nanotools and devices in probing how cells will respond to mechanical forces, microenvironment mechanics, topography, and chemical cues with the aim of elucidating molecular mechanisms of mechanotransduction in cell biology. In fact, fundamental biological processes such as apoptosis (cell death), mitosis, cancer metastasis, and stem cell fate are known to be modulated and even controlled by such mechanical cues [1, 5, 8, 10-12, 18].

Wang and Pelling [22] showed that the nanomechanical breakdown of cells within a monolayer is governed by the depolymerization of F-actin within the cells, while the extracellular matrix (ECM) maintains the integrity of the sheet. Although cells within the monolayer are experiencing massive internal cytoskeletal remodeling in early apoptosis, the ECM clearly maintains the overall stability of the cell sheet.

Qian and Wang [19] reviewed studies integrating micro/ nano fabrication work to elucidate the molecular mechanism of signaling transduction in cell biology. The different micro/nanofabrication technologies include those that can generate three-dimensional scaffolds for tissue engineering. The application of these technologies is in their ability to manipulate the physical or chemical micro/nano-environment to regulate cellular behavior and response, such as cell life and death, differentiation, proliferation, and cell migration.

As mentioned, cells are known to respond to chemical and topographic cues in their surroundings. For example, adhesion-promoting molecules such as poly-L-lysine (PLL) and fibronectin (Fn) are commonly used in cell assays to enable cell spreading and proliferation and to promote biocompatibility of biomedical devices. Similarly, engineered topography is often used to guide cell growth and differentiation. However, little is known about how these cues affect the biomechanical properties of cells and subsequent cell function. McPhee et al. [15] applied atomic force microscopy to investigate and quantify how PLL, Fn, and microtopography can affect cellular elasticity and the organization of the cytoskeleton. They hope their work can shed light on whether engineered chemical cues can eventually be masked by a cell's own matrix proteins and so only exert short-term influence, and whether topographical and chemical cues both affect cell elasticity.

Cell-substrate interactions can affect cellular functions such as spreading, migration, and even differentiation. These interactions are often studied using micro- and nanotechnological tools. The design of substrates based on these technologies offers new ways to probe cellular responses to changes in their physical environment. In fact, this allows the interactions of cells and their surrounding matrix to be carried out in well-controlled conditions. Here, Digabel et al. [7] reviewed techniques and tools based on nano- and microfabrication to analyze the influence of the mechanical properties of the substrate on cell functions such as cell adhesion and migration and how these can lead to better understanding of cell mechanobiology.

Cell membranes exhibit complexity which ranges from their molecular contents to their emergent mechanical 
properties and dynamic spatial organization. These compositional and geometrical organizations of membrane components are known to influence cell functions such as signal transduction. Supported membranes, consisting of a bilayer assembly of phospholipids on a solid substrate, have been used as model systems to study a wide range of problems in cell biology [3,4]. The spatial organization and mechanical deformation of supported membranes can also be manipulated by patterning underlying substrates with micro and nanofabrication techniques. Yu and Groves [23] presented various applications and methods to spatially patterned biomembranes by means of curvature modulations and spatial reorganizations, and utilizing them to interface with living cells. This unique approach allows one to investigate mechanisms of interfacial interaction and signaling in cell biology.

Finally, efficient cell separation is an essential first step in many biological and medical assays and also for disease detection and diagnosis. This need has led to the development of numerous micro-separation techniques. Bhagat et al. [2] reviewed the current state-of-the-art in microfluidics-based cell separation techniques. The various separation principles are explained along with examples demonstrating their application in cell separation. Developing efficient microscale separation methods that offer greater control in isolating or enriching specific-targeted cells will be important in realizing true point-of-care labon-a-chip systems.

As can be seen from this special issue, these articles showcase some very innovative and exciting use of micro and nanotechnology in understanding cell and molecular biology as well as in detecting and diagnosing diseases. While some are still very much at the research stage, there is no doubt that enormous progress will be made in the coming years, and it is hope that some of the important findings arising from these research will result in actual biomedical applications in the not too distant future.

\section{References}

1. Alenghat FJ, Ingber DE (2002) Mechanotransduction: All signals point to cytoskeleton, matrix, and integrins. Sci STKE:PE6

2. Bhagat AAS, Bow H, Hou HW, Tan SJ, Han J, Lim CT (2010) Microfluidics for cell separation. Med Biol Eng Comput. doi: 10.1007/s11517-010-0611-4

3. Brian AA, McConnell HM (1984) Allogeneic stimulation of cytotoxic T cells by supported planar membranes. Proc Natl Acad Sci USA 81:6159-6163

4. Chan YH, Boxer SG (2007) Model membrane systems and their applications. Curr Opin Chem Biol 11:581-587
5. Chicurel ME, Chen CS, Ingber DE (1998) Cellular control lies in the balance of forces. Curr Opin Cell Biol 10(2):232-239

6. Chin CD, Linder V, Sia SK (2007) Lab-on-a-chip devices for global health: past studies and future opportunities. Lab Chip 7(1):41-57

7. Digabel JL, Ghibaudo M, Trichet L, Richert A, Ladoux B (2010) Microfabricated substrates as a tool to study cell mechanotransduction. Med Biol Eng Comput. doi:10.1007/s11517-010-0619-9

8. Engler AJ, Sen S, Sweeney HL, Discher DE (2006) Matrix elasticity directs stem cell lineage specification. Cell 126(4): $677-689$

9. Gardeniers JGE, van den Berg A (2004) Lab-on-a-chip systems for biomedical and environmental monitoring. Anal Bioanal Chem 378(7):1700-1703

10. Hogan C, Dupre-Crochet S, Norman M, Kajita M, Zimmermann C, Pelling AE, Piddini E, Baena-Lopez LA, Vincent JP, Itoh Y, Hosoya H, Pichaud F, Fujita Y (2009) Characterization of the interface between normal and transformed epithelial cells. Nat Cell Biol 11:460-467

11. Huang S, Ingber DE (2005) Cell tension, matrix mechanics, and cancer development. Cancer Cell 8:175-176

12. Kunda P, Pelling AE, Liu T, Baum B (2008) Moesin controls cortical rigidity, cell rounding, and spindle morphogenesis during mitosis. Curr Biol 18:91-101

13. Llandro J, Palfreyman JJ, Ionescu A, Barnes CHW (2010) Magnetic biosensor technologies for medical applications: a review. Med Biol Eng Comput. doi:10.1007/s11517-010-0649-3

14. Mauritz JMA, Esposito A, Tiffert T, Skepper JN, Warley A, Yoon Y-Z, Cicuta P, Lew VL, Guck JR, Kaminski CF (2010) Biophotonic techniques for the study of malaria-infected red blood cells. Med Biol Eng Comput. doi:10.1007/s11517-0100668-0

15. McPhee G, Dalby MJ, Riehle M, Yin H (2010) Can common adhesion molecules and microtopography affect cellular elasticity? A combined atomic force microscopy and optical study. Med Biol Eng Comput. doi:10.1007/s11517-010-0657-3

16. Nagarajan S, Li Z, Marchi-Artzner V, Grasset F, Zhang Y (2010) Imaging gap junctions with silica-coated upconversion nanoparticles. Med Biol Eng Comput. doi:10.1007/s11517-010-0618-x

17. Pankhurst QA, Thanh NKT, Jones SK, Dobson J (2009) Progress in application of magnetic nanoparticles in biomedicine. J Phys D 42:224001

18. Pelling AE, Veraitch FS, Chu CPK, Mason C, Horton MA (2009) Mechanical dynamics of single cells during early apoptosis. Cell Motil Cytoskeleton 66(7):409-422

19. Qian T, Wang Y (2010) Micro/nano-fabrication technologies for cell biology. Med Biol Eng Comput. doi:10.1007/s11517-0100632-z

20. Soper SA et al (2006) Point-of-care biosensor systems for cancer diagnostics/prognostics. Biosens Bioelectron 21(10):1932-1942

21. Tudos AJ, Besselink GAJ, Schasfoort RBM (2001) Trends in miniaturized total analysis systems for point-of-care testing in clinical chemistry. Lab Chip 1(2):83-95

22. Wang J, Pelling AE (2010) Cell sheet integrity and nanomechanical breakdown during programmed cell death. Med Biol Eng Comput. doi:10.1007/s11517-010-0640-z

23. Yu C, Groves JT (2010) Engineering supported membranes for cell biology. Med Biol Eng Comput. doi:10.1007/s11517010-0634-x 